\title{
Erratum to: Revision of Isanodus paladeji (Elasmobranchii, Hybodontiformes) from the Lower Cretaceous of Thailand
}

\author{
Suchada Khamha ${ }^{1} \cdot$ Gilles Cuny $^{2} \cdot$ Komsorn Lauprasert $^{1}$
}

Published online: 12 September 2016

(c) Paläontologische Gesellschaft 2016

\section{Erratum to: Paläontol $\mathrm{Z}$ \\ DOI 10.1007/s12542-015-0282-4}

In the original article the German abstract ("Kurzfassung") contained printing errors. The correct "Kurzfassung" is reproduced in full below. We apologize for this error.

Kurzfassung Mikrofossilien aus der Unter-Kreide von Phu Phan Thong (Provinz Nong Bua Lam) in Thailand lieferten zehn Zahn-Morphotypen der Süßwasser-Haigattung Isanodus, die eine Revision dieser Gattung erlauben. Zwei neue Morphotypen besitzen gemeinsame Merkmale mit den vorderen und hinteren Zähnen von Isanodus paladeji und werden als anterolaterale oder posterolaterale Zähne betrachtet. Die Zähne, die früher als anterolaterale oder posterolaterale Zähne von I. paladeji identifiziert wurden, scheinen in der Tat zu einer neuen Art, Isanodus nongbualamphuensis sp. nov., zu gehören. Das Ornamentationsmuster einiger Zähne von I. paladeji und I. nongbualamphuensis sp. nov. ist dem der Gattung Heteroptychodus ziemlich ähnlich, was eine enge Verwandtschaft dieser beiden Gattungen andeutet.

The online version of the original article can be found under doi:10.1007/s12542-015-0282-4.

Komsorn Lauprasert

lauprasert@gmail.com

1 Department of Biology, Faculty of Science, Mahasarakham University, Khamrieng, Kantharawichai District,

Maha Sarakham 44150, Thailand

2 UMR CNRS 5276 LGLTPE ENS, Université Claude Bernard Lyon 1, 2, rue Raphaël Dubois, 69622 Villeurbanne Cedex, France 